\title{
Influencia del estado nutricional, niveles hormonales séricos e historia familiar de cáncer en el desarrollo del cáncer de mama
}

\author{
EMILIO GONZÁLEZ JIMÉNEZ1', PEDRO A. GARCÍA LÓPEZ², \\ JACQUELINE SCHMIDT-RÍO-VALLE ${ }^{3}$, CARMEN VALENZA ${ }^{4}$
}

\section{Influence of nutritional status, hormones serum levels, and family history on breast cancer development}

Background: Several studies have analyzed the relation between obesity and the hormonal imbalances generated by overweight and a family history of breast cancer. All of these factors are potentially implicated in the early development of breast cancer. Aim: To verify the existence of a significant relation between the nutritional status of breast cancer patients, their hormone serum levels (estrogens, prolactin, and progesterone), and the existence of a family history of breast cancer. Material and Methods: Retrospective data was collected from clinical records of 524 women diagnosed with breast cancer in a Spanish hospital. Results: There was a positive association between estrogen, progesterone and prolactin serum levels and body mass index. The elevations in hormone levels occurred earlier in life among women with a family history of breast cancer. A two way ANOVA found a significant association between progesterone and prolactin levels with the age at diagnosis of breast cancer. Conclusions: Extreme serum levels of these hormones appear to be related to the early development of breast cancer, which in turn is influenced by the existence of a family history of cancer among those women with normal or average hormone levels.

(Rev Med Chile 2012; 140: 1263-1267).

Key words: Breast neoplasms; Menarche; Obesity.

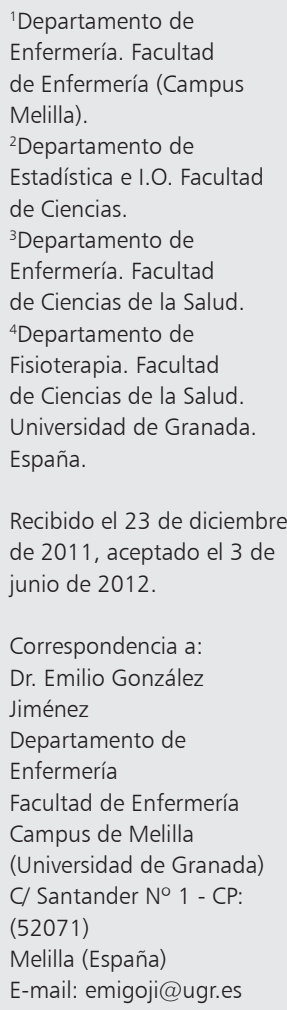

$\mathrm{D}$ iferentes estudios han intentado verificar una posible relación entre la obesidad y el desarrollo precoz de ciertos tipos de cáncer como el de mama ${ }^{1}$. En el caso de los tumores malignos de mama, la existencia o no de antecedentes familiares de cáncer de mama, constituye un factor relevante a tener en cuenta $^{2,3}$. Así, según datos del estudio desarrollado por Lancaster $(2005)^{4}$, hasta $18 \%$ de las mujeres obesas con cáncer de mama tenían antecedentes familiares de este tipo de cáncer. De acuerdo con Maheu y cols $(2008)^{5}$ y Qiu y cols $(2010)^{6}$ serían las mutaciones de los genes $B R C A-1$ y $B R C A-2$ las responsables de gran parte de los casos con patrón hereditario familiar.
No obstante, los desórdenes metabólicos y hormonales secundarios a estados de obesidad parecen ser los responsables últimos del incremento en la prevalencia de neoplasias mamarias malignas en mujeres obesas ${ }^{7,8}$. Según esto y de acuerdo con Veronesi y cols $(2005)^{9}$, el incremento de adiposidad corporal tan necesario para la llegada de la primera regla así como la elevación de los niveles hormonales que de ello resultan, van a constituir factores de riesgo cruciales para el desarrollo precoz de cáncer de mama entre mujeres. En este sentido, el riesgo de padecer cáncer de mama se multiplica para aquellas mujeres que a edades tempranas se muestran obesas ${ }^{10}$.

Con respecto a la acción de dichas hormonas, 
se ha descrito una asociación estadísticamente significativa entre niveles séricos elevados de estrógenos en mujeres obesas y el desarrollo precoz del cáncer de mama ${ }^{11}$. Si bien, existen controversias sobre si esta misma cuestión sucede de igual forma con hormonas como la prolactina o la progesterona $^{12,13}$.

Las mujeres obesas poseen un riesgo mayor de padecer cáncer de mama después de la menopausia comparado con aquellas mujeres no obesas ${ }^{14}$. Esto parece tener su explicación en los altos niveles de estrógenos circulantes en las mujeres obesas ${ }^{14}$. En aquellas mujeres obesas posmenopáusicas los niveles de estrógenos son entre 50-100\% más elevados que entre las mujeres delgadas ${ }^{15}$. No debemos olvidar que antes de la menopausia, los ovarios son la fuente principal de estrógenos aunque también el tejido adiposo. Después de la menopausia, los ovarios dejan de producir estrógenos convirtiéndose pues el tejido adiposo en la principal fuente de esta hormona. Luego aquellos tejidos que como el parénquima mamario son muy sensibles a los estrógenos, quedan expuestos a un mayor estímulo hormonal en las mujeres obesas ${ }^{16}$. Esta circunstancia lleva a un riesgo mayor de desarrollar una neoplasia y en su caso a un crecimiento más rápido de tumores hormono-dependientes, fundamentalmente de los estrógenos, progesterona y prolactina ${ }^{17}$. Teniendo en consideración todo lo anterior, el objetivo de este trabajo fue comprobar la existencia de una relación significativa entre el estado nutricional de las pacientes, sus niveles séricos de estrógenos, progesterona y prolactina, la existencia o no de antecedentes familiares de cáncer de mama y cómo todo ello influye en un desarrollo precoz del cáncer de mama.

\section{Objetivo}

El objetivo de este trabajo fue comprobar la existencia de una relación significativa entre el estado nutricional de las pacientes, sus niveles séricos hormonales (estrógenos, prolactina y progesterona), edad de diagnóstico y la existencia o no de antecedentes familiares de cáncer de mama.

\footnotetext{
Muestra

La muestra estaba compuesta por 524 pacientes de sexo femenino, diagnosticadas y tratadas de cáncer de mama en el Hospital Universitario "San Cecilio" de Granada, durante el período de enero de 2009 a septiembre de 2010 .
}

\section{Metodología}

Se llevó a cabo una recogida de datos retrospectiva de todas y cada una de las pacientes por medio de una revisión minuciosa de su historia clínica. El análisis posterior de los datos fue realizado con el programa informático $\mathrm{R}$ (versión 2.13.2) ${ }^{18}$.

\section{Resultados}

En relación con las hormonas analizadas (Figura 1), los niveles de estrógenos circulantes mantienen una relación estrecha con el estado nutricional de las pacientes, esto es, a medida que las pacientes se hacían obesas, los niveles séricos de estrógenos se elevaban. Asimismo, la existencia de antecedentes familiares de cáncer resultó ser un factor implicado, esto es, los niveles séricos de estrógenos se elevaban más tempranamente en edad entre las pacientes que presentaban antecedentes familiares de cáncer de mama. Si bien, las mayores diferencias se encontraron en el grupo de mujeres con obesidad mórbida, con unos niveles circulantes de estrógenos muy elevados a cortas edades así como una edad de diagnóstico del tumor temprana. Respecto de la variable progesterona, los resultados muestran que sus valores séricos se incrementaban en estados de obesidad y obesidad mórbida. Al igual que con los estrógenos, los niveles de progesterona eran más elevados a edades más tempranas entre el colectivo de pacientes con antecedentes familiares de cáncer de mama. Finalmente, en el caso de la hormona prolactina, sus valores se incrementaban a medida que las pacientes se hacían obesas, siendo especialmente temprana en edad su elevación entre el grupo con antecedentes familiares de cáncer.

Mediante un análisis ANOVA a dos vías con los factores obesidad (pacientes no obesas, obesas y obesas mórbidas), historia familiar de cáncer (pacientes sin antecedentes familiares de cáncer y con antecedentes), y para las variables respuesta (edad de diagnóstico, estradiol, progesterona y prolactina no se encontraron interacciones en los factores para edad de diagnóstico $(\mathrm{F}=1,1615$; $\mathrm{p}=0,313818)$ y estradiol $(\mathrm{F}=1,7653 ; \mathrm{p}=0,1722)$ y sí para progesterona $(F=5,0122 ; p=0,006984)$ y prolactina $(\mathrm{F}=3,9377 ; \mathrm{p}=0,02008)$. Ahora bien, para el factor obesidad, todas las categorías contempladas mostraron diferencias estadísticamente 


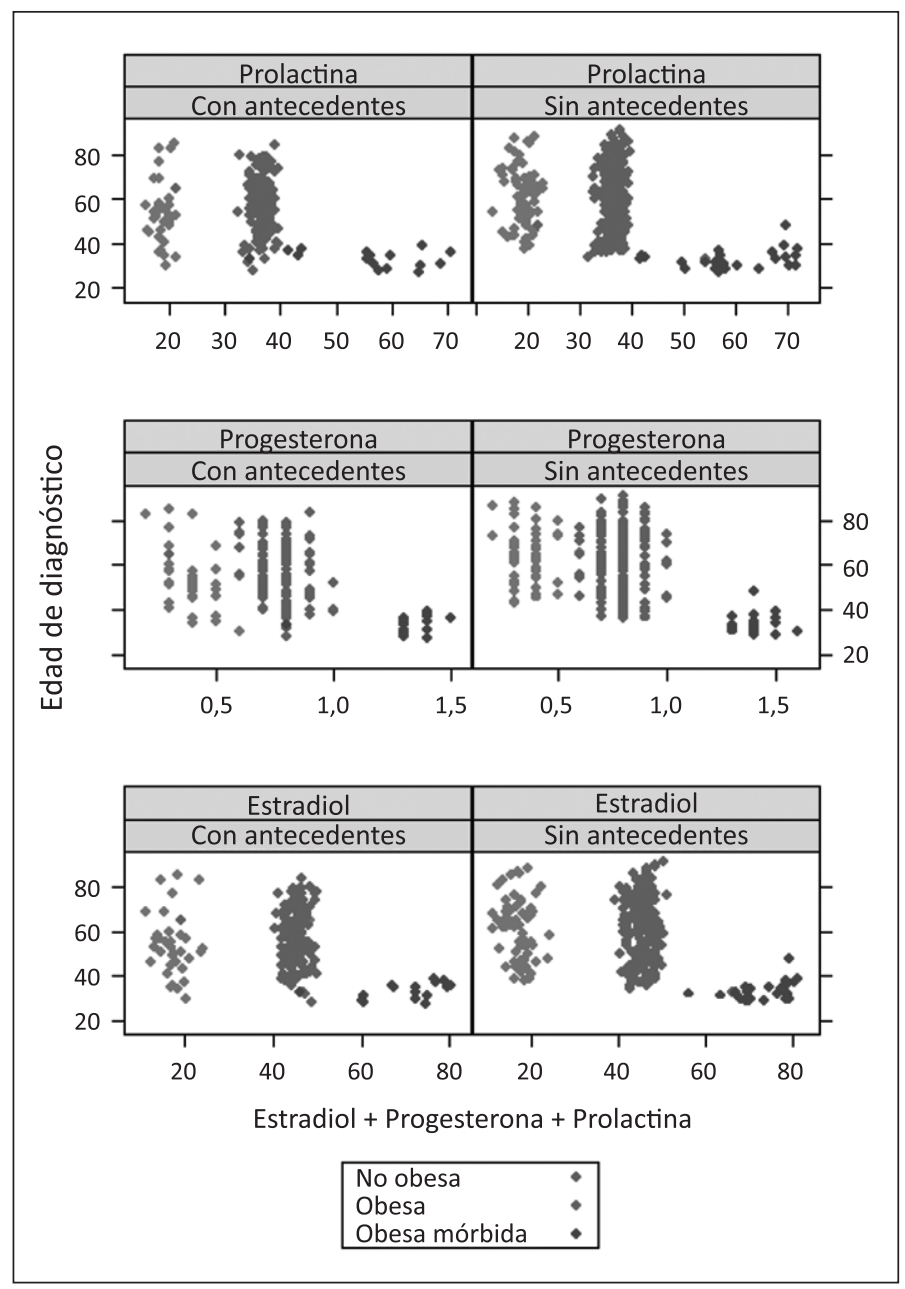

Figura 1. Correlación entre estado nutricional, edad de diagnóstico, niveles séricos hormonales y antecedentes familiares o no para cáncer de mama. significativas, salvo para la variable edad de diagnóstico, en cuyo caso, el nivel de significación sería atribuible al grupo de obesas mórbidas. Asimismo, para esta variable se observan diferencias en el factor historia familiar de cáncer. Para el resto de variables, el factor historia no resultó ser significativo, como cabía esperar. Estos resultados se muestran en la Tabla 1 .

\section{Discusión}

De acuerdo con nuestros resultados, niveles elevados de estas hormonas en mujeres con obesidad y obesidad mórbida se asocian a una edad más temprana de diagnóstico de cáncer mamario. Estos resultados contrastan con los obtenidos en otros estudios en los que no se encontró una asociación entre el estado nutricional de las pacientes, los niveles hormonales y en consecuencia una edad de diagnóstico más temprana ${ }^{19,20,21}$.

Otros autores como Perks et al., 201122; Bradlow et al., 2011 23 y McTiernan et al., 2006 ${ }^{24}$, coinciden al encontrar una asociación directa y significativa entre la obesidad, niveles séricos hormonales y un desarrollo precoz del cáncer mamario. Esta relación podría tener su explicación

Tabla 1. Variables analizadas y niveles de significación

\begin{tabular}{|c|c|c|c|c|c|c|c|c|c|}
\hline \multirow{3}{*}{$\begin{array}{c}\begin{array}{c}\text { Historia familiar } \\
\text { de cáncer }\end{array} \\
\text { Sí } \\
\text { No }\end{array}$} & \multicolumn{2}{|c|}{ No obesas } & \multicolumn{2}{|c|}{ Obesas } & \multicolumn{2}{|c|}{$\begin{array}{l}\text { Obesidad } \\
\text { mórbida }\end{array}$} & \multirow{3}{*}{$\begin{array}{l}\text { Respuesta } \\
\text { Edad de diagnóstico }\end{array}$} & \multirow{3}{*}{$\begin{array}{c}\begin{array}{c}\text { Factor } \\
\text { obesidad }\end{array} \\
\begin{array}{c}\mathrm{F}=1788,1 \\
\mathrm{p}<0,0001\end{array}\end{array}$} & \multirow{3}{*}{$\begin{array}{c}\begin{array}{c}\text { Factor } \\
\text { historia }\end{array} \\
\mathrm{F}=9,9464 \\
\mathrm{p}=0,0017\end{array}$} \\
\hline & 54,1 & $(13,7)$ & 57,2 & $(12,3)$ & 33,3 & $(3,6)$ & & & \\
\hline & 60,5 & $(14,0)$ & 60,8 & $(14,5)$ & 32,1 & $(5,3)$ & & & \\
\hline Sí & 18,2 & $(6,1)$ & 45,3 & $(2,9)$ & 70,0 & $(9,2)$ & \multirow{2}{*}{ Estradiol } & \multirow{2}{*}{$\begin{array}{l}F=3518,9 \\
p<0,0001\end{array}$} & \multirow{2}{*}{$\begin{array}{l}F=0,7893 \\
p=0,3747\end{array}$} \\
\hline No & 17,2 & $(2,8)$ & 45,8 & $(2,6)$ & 71,4 & $(7,5)$ & & & \\
\hline Sí & 0,41 & $(0,11)$ & 0,77 & $(0,09)$ & 1,32 & $(0,15)$ & \multirow{2}{*}{ Progesterona } & \multirow{2}{*}{$\begin{array}{l}F=1788,1 \\
p<0,0001\end{array}$} & \multirow{2}{*}{$\begin{array}{l}F=2,1178 \\
p=0,1462\end{array}$} \\
\hline No & 0,38 & $(0,09)$ & 0,78 & $(0,09)$ & 1,40 & $(0,08)$ & & & \\
\hline Sí & 19,4 & $(3,1)$ & 36,6 & $(1,9)$ & 56,1 & $(10,5)$ & \multirow{2}{*}{ Prolactina } & \multirow{2}{*}{$\begin{array}{l}F=2003,0 \\
p<0,0001\end{array}$} & \multirow{2}{*}{$\begin{array}{l}F=1,1392 \\
p=0,2863\end{array}$} \\
\hline No & 19,4 & $(2,9)$ & 36,7 & $(2,1)$ & 59,4 & $(9,1)$ & & & \\
\hline
\end{tabular}


Estado nutricional, niveles hormonales séricos e historia familiar en cáncer de mama - E. González Jiménez et al

en la teoría por la cual se concede fundamentalmente a los estrógenos una potencial capacidad carcinogénica a través de la continua estimulación del crecimiento tisular mamario ${ }^{24}$. Luego el efecto de la obesidad sobre el desarrollo cada vez más precoz de cáncer de mama en mujeres, podría tener su fundamento en el subsiguiente incremento de hormonas circulantes, especialmente de estradiol ${ }^{25}$. Que la obesidad constituye un factor de riesgo estrechamente relacionado con la edad de diagnóstico del cáncer de mama supone un hecho que trasciende de otros factores como pueden ser la existencia o no de antecedentes familiares para cáncer de mama. No obstante, en este estudio los antecedentes familiares de cáncer considerados, fueron únicamente los de primer grado. Si bien, a pesar de la indudable importancia del componente genético en esta neoplasia, en nuestro estudio resultaron ser la obesidad y los niveles elevados de prolactina, progesterona y estrógenos fundamentalmente, los factores más implicados en el desarrollo precoz del cáncer de mama $^{26}$. En cualquier caso, y con independencia de los hallazgos descritos resulta indispensable continuar profundizando y analizando las múltiples causas y factores de potencial implicación en el cáncer de mama.

\section{Referencias}

1. Amaral P, Miguel R, Mehdad A, Cruz C, Monteiro-Grillo I, Camilo M, et al. Body fat and poor diet in breast cancer women. Nutr Hosp 2010; 25 (3): 456-61.

2. Alhurishi S, Lim JN, Potrata B, West R. Factors influencing late presentation for breast cancer in the middle East: a systematic review. Asian Pac J Cancer Prev 2011; 12 (6): 1597-600.

3. Razif SM, Sulaiman S, Hanie SS, Aina EN, Rohaizak M, Fuad I, et al. The contribution of reproductive factors and family history towards premenopausal breast cancer risk in Kuala Lumpur, Malaysia. Med J Malaysia 2011; 66 (3): 220-6.

4. Lancaster DR. Coping with Appraised Breast Cancer Risk Among Women with Family Histories of Breast Cancer. Research in Nursing \& Health 2005; 28, 144-58.

5. Maheu C, Thorne S. Receiving Inconclusive Genetic Test Results: An Interpretive Description of the BRCA1/2 Experience. Research in Nursing \& Health 2008; 31, 553-62.

6. Qiu L, Yao L, Xue K, Zhang J, Mao Ch, Chen B, et al.
BRCA2 N372H polymorphism and breast cancer susceptibility: a meta-analysis involving 44.903 subjects. Breast Cancer Res Treat 2010; 123: 487-90.

7. Zorlini R, Akemi Abe Cairo A, Salete Costa Gurgel M. Nutritional status of patients with gynecologic and breast cancer. NutrHosp 2008; 23 (6): 577-83.

8. Key TJ, Appleby PN, Reeves GK, Roddam AW, Helzlsouer KJ, Alberg AJ, et al. Circulating sex hormones and breast cancer risk factors in postmenopausal women: reanalysis of 13 studies. Br J Cancer 2011; 105 (5): 709 22.

9. Veronesi U, Boyle P, Goldhirsch A, Orecchia R, Viale G. Breast cancer. Lancet 2005; 365 (9472): 1727-41.

10. Di Pietro PF, Medeiros NI, Vieira FGK, Fausto MA, Belló-Klein A. Breast cancer in southern Brazil: association with past dietary intake. NutrHosp 2007; 22 (5): 565-72.

11. Key TJ. Endogenous oestrogens and breast cancer risk in premenopausal and postmenopausal women. Steroids 2011; 76 (8): 812-5.

12. Fernández I, Touraine P, Goffin V. Prolactin and human tumourogenesis. J Neuroendocrinol 2010; 22 (7): 771-7

13. Lange CA, Yee D. Progesterone and breast cancer. Womens Health 2008; 4 (2): 151-62

14. Yoshimoto N, Nishiyama T, Toyama T, Takahashi S, Shiraki N, Sugiura H, et al. Genetic and environmental predictors, endogenous, hormones and growth factors, and risk of estrogen receptor positive breast cancer in Japanese women. Cancer Sci 2011; 26 [Epub ahead of print]

15. Li XJ, Wei B, Chen HJ, Zhang Z, Zhang HY, Bu H. Heatinduced antigen retrieval in inmunohistochemistry for estrogen and progesterone receptors in breast cancer tissues. Zhonghua Bing Li XueZaZhi 2011; 40 (6): 406-8.

16. Beral V. Breast cancer and hormone-replacement therapy in the Million Women Study. Lancet 2003; 362 (9382): 419-27.

17. Faupel-Badger JM, Sherman ME, García Closas M, Gaudet MM, Falk RT, Andaya A, et al. Prolactin serm levels and breast cancer: relationships with factors and tumour characteristics among pre- and postmenopausal women in a population-based case-control study from Poland. Br J Cancer 2010; 103 (7): 1097-102.

18. R Development Core Team. R: A language and environment for statistical computing. R Foundation for Statistical Computing, Vienna, Austria, 2011. URL http:// www.R-project.org/.

19. Yager JD, Davidson NE. Estrogen carcinogenesis in breast cancer. N Engl J Med 2006; 354 (3): 270-82.

20. Tehard B, Clavel-Chapelon F. Several anthropometric measurements and breast cancer risk: results of the E3N 
cohort study. Int J Obes (Lond) 2006; 30: 156-63.

21. Sonnenschein E, Toniolo P, Terry MB, Bruning PF, Kato I, Koening KL. Body fat distribution and obesity in premenopausal and postmenopausal breast cancer. Int J Epidemiol 1999; 28: 1026-31.

22. Perks CM, Holly JM. Hormonal mechanisms underlying the relationship between obesity and breast cancer. Endocrinol Metab Clin North Am 2011; 40 (3): 485-507.

23. Bradlow HL, Sepkovic DW, Telang N, Tiwari R. Adipocyte-derived factor as a modulator of oxidative estrogen metabolism: implications for obesity and estrogendependent breast cancer. In Vivo 2011; 25 (4): 585-8.

24. McTiernan A, Wu L, Chen C, Chlebowski R, MossavarRahmani Y, Modugno F, et al. Women's Health Initiative Investigators. Relation of BMI and physical activity to sex hormones in postmenopausal women. Obesity 2006;
14 (9): 1662-77.

25. James RE, Lukanova A, Dossus L, Becker S, Rinaldi S, Tjønneland A, et al. Postmenopausal serum sex steroids and risk of hormone receptor -positive and -negative breast cancer: a nested case-control study. Cancer Prev Res (Phila) 2011; 4 (10): 1626-35.

26. Rossouw JE, Anderson GL, Prentice RL, LaCroix AZ, Kooperberg C, Stefanick ML, et al. Risks and benefits of estrogen plus progestin in healthy postmenopausal women: principal results From the Women's Health Initiative randomized controlled trial. JAMA 2002; 288 (3): 321-33.

27. Aguilar Cordero MJ, González Jiménez E, Álvarez Ferre J, Padilla López CA, Mur Villar N, García López PA, et al. Lactancia materna: un método eficaz en la prevención del cáncer de mama. Nutr Hosp 2010; 25 (6): 954-8. 